\title{
Acute gastrointestinal injury in the intensive care unit: a retrospective study
}

This article was published in the following Dove Press journal:

Therapeutics and Clinical Risk Management

5 October 2015

Number of times this article has been viewed

\author{
HuaiSheng Chen ${ }^{1, *}$ \\ HuaDong Zhang ${ }^{1, *}$ \\ Wei $\mathrm{Li}^{\prime}$ \\ ShengNan $\mathrm{Wu}^{\prime}$ \\ Wei Wang ${ }^{2}$
}

'Intensive Care Unit, ${ }^{2}$ Endocrinology Department, Second Affiliated

Hospital of Jinan University, Shenzhen

People's Hospital, Shenzhen,

People's Republic of China

*These authors contributed equally to this work
Correspondence: Wei Wang Endocrinology Department, Second Affiliated Hospital of Jinan University, Shenzhen People's Hospital, 1017

Dong Men North Road, Luohu District, Shenzhen 518020, Guangdong,

People's Republic of China

Tel +8675525533018

Fax +867552553 3497

Email wangwszhen@yeah.net
Background: Acute gastrointestinal injury (AGI) is a common problem in the intensive care unit (ICU). This study is a review of the gastrointestinal function of patients in critical care, with the aim to assess the feasibility and effectiveness of grading criteria developed by the European Society of Intensive Care Medicine (ESICM) Working Group on Abdominal Problems (WGAP).

Methods: Data of patients who were admitted to the ICU of Shenzhen People's Hospital, Shenzhen, People's Republic of China, from January 2010 to December 2011 were reviewed. A total of 874 patients were included into the current study. Their sex, age, ICU admissive causes, complication of diabetes, AGI grade, primary or secondary AGI, mechanical ventilation (MV), and length of ICU stay (days) were recorded as risk factors of death. These risk factors were studied by unconditioned logistic regression analysis.

Results: All the risk factors affected mortality rate. Unconditional logistic regression analysis revealed that the mortality rate of secondary AGI was 71 times higher than primary AGI (odds ratio [OR $] 4.335,95 \% \mathrm{CI}[1.652,11.375])$. When the age increased by one year, the mortality probability would increase fourfold. Mortality in patients with MV was 63-fold higher than for patients with non-MV. Mortality rate increased 0.978 times with each additional day of ICU stay.

Conclusion: Secondary AGI caused by severe systemic conditions can result in worsened clinical outcomes. The 2012 ESICM WGAP AGI recommendations were to some extent feasible and effective in guiding clinical practices, but the grading system lacked the support of objective laboratory outcomes.

Keywords: critical care, acute gastrointestinal injury, mortality

\section{Introduction}

Acute gastrointestinal injury (AGI) is a common problem in intensive care units (ICUs), and it is usually caused by multiple factors. ${ }^{1}$ Critical illness can cause gastrointestinal peristalsis disorders ${ }^{2}$ and intestinal mucosal ischemia, resulting in intestinal contraction and gut barrier function damage. At the same time, the presence of systemic inflammatory reaction syndrome and multiple organ dysfunction syndrome (MODS) can also cause AGI. ${ }^{3}$ Mortality rate is increased by inflammatory reactions, due to the ectopic intestinal flora. ${ }^{4}$ Insufficient understanding and definition of AGI in the past has led to the lack of detection methods and less clinical trials, and therefore, no guidelines for treating AGI have been developed. Various definitions and grades have, however, been proposed in previous clinical research.

In the year 2012, the European Society of Intensive Care Medicine (ESICM) Working Group on Abdominal Problems (WGAP) developed a recommendation on AGI, which defined and graded AGI as a critical illness. ${ }^{5}$ The ESICM is an association of individual persons. ${ }^{5}$ The ESICM was founded in March 1982 in Geneva, Switzerland. ESICM supports and promotes the advancement of knowledge in intensive care 
medicine, in particular the promotion of the highest standards of multidisciplinary care of critically ill patients and their families through education, research, and professional development. ${ }^{5}$

We reviewed partial records of patients with critical illness, re-grouped these patients under the ESICM WGAP grades of AGI, and compared the outcomes of these patients, to assess the feasibility and effectiveness of AGI recommendation on clinical practice. ${ }^{5}$

\section{Materials and methods Inclusive population}

Records of patients who were admitted to the ICU of the Shenzhen People's Hospital from January 2010 to December 2011 were reviewed. There were a total of 925 cases, among which ten patients were readmitted more than once. Patients readmitted to the ICU once were calculated as one case, while patients who were readmitted to the hospital several times were calculated as several cases according to the number of hospital admissions. Finally, 874 cases were evaluated in our current study (51 cases were excluded).

\section{Therapeutic protocols}

Patients were treated according to clinical guidelines of the second affiliated hospital of Jinan University with reference to different causes. Broad-spectrum antibiotics were applied to septic patients. For severe sepsis or septic shock, bundle therapeutics were given. For patients with MODS, microcirculation of organs and tissues was maintained, based on organ supportive measures. A calorie-count diet of $20 \mathrm{kcal} / \mathrm{kg}$ body weight per day was administered when a patient was in a critical state, and calorie count was increased gradually if the disorder or condition improved.

\section{Outcome measurements}

AGI grades were applied according to the ESICM recommendations from 2012. ${ }^{5}$ Gastrointestinal surgery or trauma that induced AGI was defined as primary AGI, while AGI caused by systemic condition or distant organ injury was defined as secondary AGI. Clinical end point outcomes were death and ICU stay (days). Other measurements were sex, age, complication of diabetes, ICU admission causes, and mechanical ventilation (MV).

\section{Statistical analysis}

The patients were divided according to whether they were dead or not. Distribution was estimated in measurement data. Normal distribution data were described as mean \pm standard deviation $(\mathrm{m} \pm \mathrm{SD})$ and were analyzed with the independent sample $t$-test. Non-normal distribution data were described with average numbers, which were analyzed with the MannWhitney $U$ statistic. The chi-square $\left(\chi^{2}\right)$ test was applied to count data. Death was calculated as a result variable; other measurements including sex, age, complication of diabetes, ICU admission causes, MV, AGI grade (including without AGI), and ICU length of stay (days) were analyzed as risk factors. Interactions between two factors were also introduced for discovering superposed effects between factors. Causes for admission to ICU were reduced to 14 causes, classified according to random variables. Dummy variables were set, and the data were analyzed with group-designed non-conditional logistic regression analysis. Odds ratios (ORs) and 95\% confident intervals (CIs) were calculated. $P$-values for differences between groups of $<0.05$ were considered as statistically significant. SPSS version 17.0 software was used for analysis.

\section{Results}

\section{General information}

A total of 874 cases were studied. Among them, 423 cases were male, while 451 were female. Age of patients/ cases ranged from 7 to 101 years, with an average age of $58 \pm 24$ years. Acute Physiology and Chronic Health Evaluation II (APAHCE II) score ranged from 6 to 45,

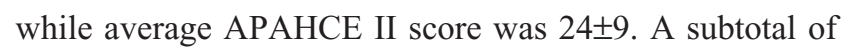
159 patients were dead, accounting for $18.2 \%$ of the total. Fourteen ICU admission causes were accounted for in this study. According to the grade of the AGI recommendation, 471 cases without AGI accounted for $53.89 \%$ of admissions, among which 19 were dead, and the mortality rate was $4.03 \%$ for non-AGI admissions. A subtotal of 403 cases had AGI, an incidence rate of $46.11 \%$. A subtotal of 140 patients died, and the mortality rate was $34.74 \%$ among AGI patients. Patients with AGI were grouped according to their AGI severity. A subtotal of 118 cases were diagnosed with AGI grade I. Six patients among them died. A subtotal of 180 cases were diagnosed with AGI grade II, among which 54 patients died. A further 76 cases were diagnosed with AGI grade III, among which 54 patients died. Finally, 29 cases were diagnosed with AGI grade IV, among which 26 died. Patient information is listed in Table 1, and mortality corresponding to AGI grade is illustrated in Figure 1.

\section{Logistic regression}

Death was considered as a result variable. Sex, age, complication of diabetes, ICU admission causes, MV, AGI grade (including without AGI and AGI grades I-IV), and ICU length 
Table I Included patients' general information

\begin{tabular}{|c|c|c|c|}
\hline & Deaths, $n$ & Survival, $\mathbf{n}$ & Statistics \\
\hline Number of cases & 159 & 715 & - \\
\hline \multicolumn{4}{|l|}{ Sex } \\
\hline Male & 95 & 328 & $\chi^{2}=10.026$ \\
\hline Female & 64 & 387 & $P=0.002$ \\
\hline \multirow[t]{2}{*}{ Age, years } & $62 \pm 20$ & $58 \pm 24$ & $t=9.413$ \\
\hline & & & $P=0.002$ \\
\hline \multicolumn{4}{|l|}{ ICU admission causes } \\
\hline Gastrointestinal carcinoma & 14 & 59 & \\
\hline Abdominal infection and non-tumor abdominal surgery & 24 & 97 & \\
\hline Obstetric pathology & 2 & 99 & \\
\hline Orthopedic surgery & I & 116 & \\
\hline Lung and bloodstream infection & 38 & 66 & \\
\hline Multiple traumas & 6 & 51 & \\
\hline Severe pancreatitis & 4 & 7 & \\
\hline Cerebrovascular accident and brain injury & 33 & 63 & \\
\hline Cardiac disorder & 8 & 15 & \\
\hline Cardiac arrest (noncardiac causes) & 10 & 6 & \\
\hline Intoxication & 4 & 2 & \\
\hline Neuromuscular disease & 0 & 5 & \\
\hline Blood system disorder & I & 5 & $\chi^{2}=128.798$ \\
\hline Other & 14 & 124 & $P<0.0001$ \\
\hline \multicolumn{4}{|l|}{ Complication of diabetes } \\
\hline Yes & 47 & 115 & $\chi^{2}=15.643$ \\
\hline No & 112 & 600 & $P<0.000$ I \\
\hline Without AGI & 19 & 452 & \\
\hline \multicolumn{4}{|l|}{ With AGI } \\
\hline Total cases & 140 & 263 & \\
\hline Grade I & 6 & 112 & \\
\hline Grade II & 54 & 126 & \\
\hline Grade III & 54 & 22 & $\chi^{2}=336.127$ \\
\hline Grade IV & 26 & 3 & $P<0.0001$ \\
\hline Primary AGI & 44 & 137 & $\chi^{2}=|60.36|$ \\
\hline Secondary AGI & 96 & 127 & $P<0.0001$ \\
\hline \multicolumn{4}{|l|}{ Mechanical ventilation } \\
\hline Yes & 158 & 369 & $\chi^{2}=123.952$ \\
\hline No & 1 & 346 & $P<0.0001$ \\
\hline \multirow[t]{2}{*}{ ICU stay (mean, days) } & 11.99 & 5.96 & $*_{z}=46,445.500$ \\
\hline & & & $P<0.000$ I \\
\hline
\end{tabular}

Notes: *Mann-Whitney $U$ statistic applied. No data denoted by hyphen.

Abbreviations: ICU, intensive care unit; AGI, acute gastrointestinal injury.

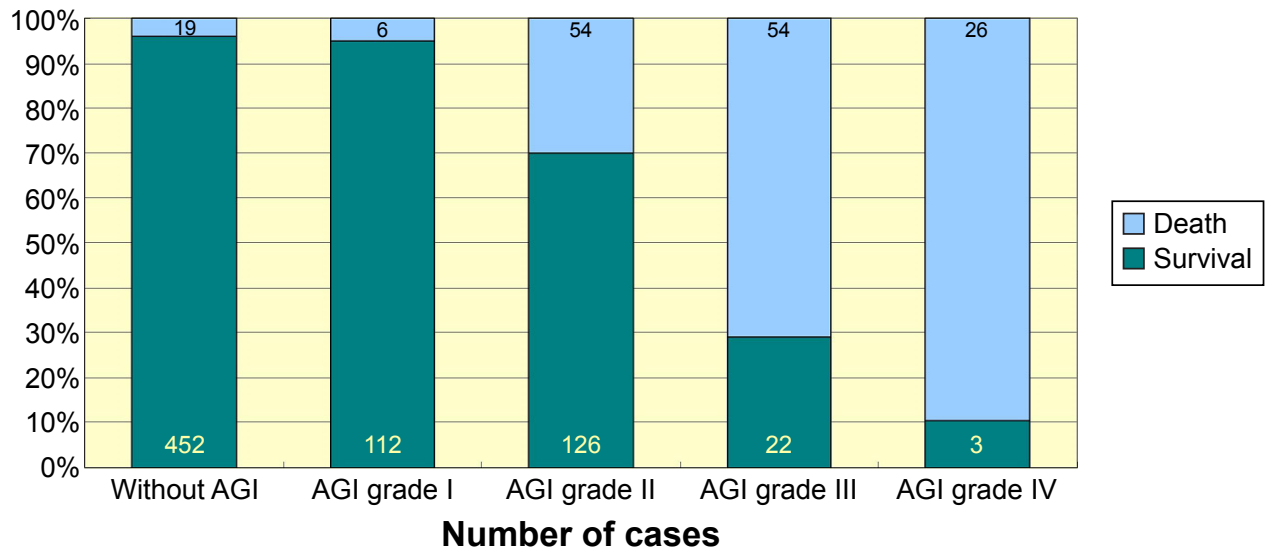

Figure I Survival and mortality rates among patients without AGI and with each grade AGI. Abbreviation: $\mathrm{AGI}$, acute gastrointestinal injury. 
Table 2 Logistic regression analysis of risk factors when death was considered as a result variable

\begin{tabular}{|c|c|c|c|c|c|c|c|c|}
\hline & \multirow[t]{2}{*}{ B } & \multirow[t]{2}{*}{ SE } & \multirow[t]{2}{*}{ Wald } & \multirow[t]{2}{*}{$d f$} & \multirow[t]{2}{*}{ Sig } & \multirow[t]{2}{*}{ OR } & \multicolumn{2}{|l|}{$95 \% \mathrm{Cl}$} \\
\hline & & & & & & & Lower & Upper \\
\hline Sex & 0.200 & 0.277 & 0.522 & I & 0.470 & 1.221 & 0.710 & 2.101 \\
\hline Age, years & 0.004 & 0.008 & 0.328 & I & 0.567 & 1.004 & 0.989 & 1.020 \\
\hline ICU admission causes & - & - & 22.881 & 13 & 0.043 & - & - & - \\
\hline Gastrointestinal carcinoma & -6.811 & 24.677 & 0.076 & 1 & 0.783 & 0.001 & 0.000 & 1.113 \\
\hline Abdominal infection and non-tumor abdominal surgery & -1.884 & 0.786 & 5.752 & 1 & 0.016 & 0.152 & 0.033 & 0.709 \\
\hline Obstetric pathology & $-1.27 \mid$ & 0.911 & 1.947 & 1 & 0.163 & 0.280 & 0.047 & 1.673 \\
\hline Orthopedic surgery & -0.200 & 0.496 & 0.163 & 1 & 0.686 & 0.819 & 0.310 & 2.162 \\
\hline Lung and bloodstream infection & -2.296 & 1.098 & 4.374 & 1 & 0.036 & 0.101 & 0.012 & 0.866 \\
\hline Multiple traumas & -0.754 & 0.724 & 1.083 & 1 & 0.298 & 0.471 & 0.114 & 1.946 \\
\hline Severe pancreatitis & -3.069 & 1.365 & 5.058 & I & 0.025 & 0.046 & 0.003 & 0.674 \\
\hline Cerebrovascular accident and brain injury & 0.670 & 0.453 & 2.186 & 1 & 0.139 & 1.954 & 0.804 & 4.746 \\
\hline Cardiac disorder & 0.120 & 0.726 & 0.027 & I & 0.869 & 1.128 & 0.272 & 4.681 \\
\hline Cardiac arrest (noncardiac causes) & 0.554 & 0.717 & 0.596 & I & 0.440 & 1.740 & 0.426 & 7.099 \\
\hline Intoxication & 1.893 & 3.275 & 0.334 & 1 & 0.563 & 6.639 & 0.011 & $4,068.956$ \\
\hline Blood system disorder & -1.100 & 0.779 & 1.996 & 1 & 0.158 & 0.333 & 0.072 & 1.531 \\
\hline Other & 0.354 & $1.56 \mathrm{I}$ & 0.052 & I & 0.820 & 1.425 & 0.067 & 30.364 \\
\hline Complication of diabetes & 0.517 & 0.331 & 2.439 & 1 & 0.118 & 1.676 & 0.877 & 3.206 \\
\hline Primary or secondary AGI & -4.237 & 1.323 & 10.249 & 1 & 0.001 & 0.014 & 0.001 & 0.193 \\
\hline Grade of $A G I$ & 1.390 & 0.163 & 72.391 & I & 0.000 & 4.016 & 2.916 & 5.532 \\
\hline MV & 4.143 & 1.049 & 15.605 & 1 & 0.000 & 62.979 & 8.063 & 491.909 \\
\hline ICU stay (mean, days) & -0.022 & 0.009 & 5.942 & 1 & 0.015 & 0.978 & 0.960 & 0.996 \\
\hline (Grade of AGI) by (primary or secondary AGI) & 1.467 & 0.492 & 8.883 & I & 0.003 & 4.335 & 1.652 & 11.375 \\
\hline Constant & -6.779 & 1.169 & 33.650 & I & 0.000 & 0.001 & - & - \\
\hline
\end{tabular}

Notes: No data denoted by hyphen. Bold indicates a statistical significance.

Abbreviations: AGI, acute gastrointestinal injury; B, regression coefficient; ICU, intensive care unit; MV, mechanical ventilation; $\mathrm{Cl}$, confidence interval; OR, odds ratio; Sig, significance; df, degrees of freedom; Wald, Wald test; SE, standard error.

of stay (days) were analyzed as risk factors. Among these risk factors, causes for admission to ICU were classified as random variables. Neuromuscular disease was used as a reference disease, as its mortality rate was $0 \%$. Other causes were referred to and compared with it in a nonconditional logistic regression analysis. Results showed that various causes induced different mortality rates $(P=0.043)$. Mortality rates of abdominal infection and non-tumor abdominal surgery, lung and bloodstream infection, and severe pancreatitis increased 0.152 -fold, 0.101-fold, and 0.046-fold, respectively, in comparison with neuromuscular disease. Mortality rate of secondary AGI was 71 times that of primary AGI, with OR 4.335, 95\% CI (1.652, 11.375). When the age increased by one year, the mortality probability would increase fourfold. Mortality in patients with MV was 63-fold higher than in patients with non-MV. Mortality rate increased 0.978 times with each additional day of ICU stay. The results of nonconditional logistic regression analysis are listed in Table 2.

\section{Discussion}

Critical care medicine focuses on patients with severe disorders, including conditions that can deteriorate into critical illness. These patients usually need ICU monitoring. ${ }^{6}$ There were 925 cases admitted in our ICU (the Shenzhen People's
Hospital, Shenzhen, People's Republic of China; 12 beds) between the years 2010 and 2011. A total of 874 cases were included into our current study, among which 159 patients died, yielding a mortality rate of $18.19 \%$. Intoxication, noncardiogenic cardiac arrest, severe pancreatitis, severe pneumonia, bloodstream infection, stroke, and severe cerebral injury had higher mortality rates. Gastrointestinal dysfunction can be a part of MODS, or an initiating factor of MODS. ${ }^{7}$ For mild AGI, feeding through trace can be affected in severe conditions. ${ }^{8}$ AGI can induce higher mortality rates in critical illness. ${ }^{9}$ From the results of our present study, we discovered that the higher grades of AGI is significantly complicated by a higher mortality rate.

All kinds of trauma, surgery, severe infection, and severe hemorrhage can promote the release of inflammatory factors, so as to activate nuclear factor KappaB signal transduction in epithelial cells in intestinal mucous membrane, ${ }^{10}$ and can result in dysfunction of its microcirculation, ${ }^{11}$ which induces histological and ultrastructural changes. Clinicians in the People's Republic of China have attributed gastrointestinal dysfunction to MODS since $1995 .{ }^{12}$ In their scoring system, abdominal bloating or decreased bowel sound is counted as 1 point, a high amount of abdominal bloating or almost disappearing bowel sound is counted as 2 points, and paralytic ileus or stress 
ulcer bleeding is counted as 3 points. As this scores system can quantify the intestinal dysfunction, it is usually applied to evaluate gastrointestinal function of critical patients in clinical practice and trials by Chinese clinicians. Abdominal bloating and decreasing bowel sounds can be caused by hypoperfusion of gastrointestinal mucosa and unrecovered intestinal injury, and both induce gradual loss of gastrointestinal secretory function and reduction of gastrointestinal hormones such as motilin and calcitonin gene-related peptide. ${ }^{13}$

However, gastrointestinal function is too complicated to be limited in its dynamic action. Intestinal bacterial translocation may be another mechanism of AGI-induced MODS. Intestinal flora can be translocated through the lymphatic system so as to activate immune reaction, ${ }^{14}$ which causes MODS or leads to further deterioration of original MODS. ${ }^{15}$ Ischemia, hypoxia, and ischemia-reperfusion injury can induce the release of inflammatory mediators that will induce intestinal dysfunction by mechanisms such as intestinal flora translocation, injury, loss of barrier function, lesions of the immune-protective system, and secretion. In turn, intestinal dysfunction reduces secreting type $\operatorname{IgA}(\mathrm{SIgA})$, and thereby negatively affects its function to locate and clear bacteria. ${ }^{16}$ All these mechanisms comprise the etiology of AGI. ${ }^{17}$

In 2012, the ESICM WGAP released recommendations that can be used to grade and quantify AGI so as to evaluate gastrointestinal function for critical-care patients. ${ }^{5} \mathrm{We}$ graded gastrointestinal function of patients admitted to the Shenzhen People's Hospital ICU from 2010 to 2011, and discovered that with the rising of grade, mortality rate was increased. The WGAP recommendations are feasible in clinical practice, but it can only be used to evaluate patients with clinical presence of AGI and reaction to therapies, without objective monitoring outcomes. In addition, some laboratory measurements can provide comprehensive understanding of gastrointestinal function. Researchers have discovered that urinary lactulose and mannitol absorption ratio, plasma D-lactate acid, endotoxin, and diamine oxidase can reflect intestinal permeability. ${ }^{18}$

According to the ESICM WGAP recommendations, AGI can be divided into primary and secondary AGI. ${ }^{5}$ Injury directly on location was defined as primary AGI, while AGI caused by worsening systemic conditions or tumorrelated injury was defined as secondary AGI. Cases with secondary AGI increased numbers in the death group in our current research. This may be due to the fact that primary AGI was primarily caused by local gastrointestinal trauma or surgery, but secondary AGI was due to deteriorating systemic disorders such as severe sepsis and shock. Therefore, secondary AGI may result in a worse prognosis. Logistic analysis further revealed that secondary AGI significantly increased mortality rate. In our present study, three causes led to a significantly worse prognosis, namely 1) nontumor abdominal infection or surgery, 2) pneumonia or bloodstream infection, and 3) severe pancreatitis. We separated severe pancreatitis from abdominal infection in our analysis, even though in the early stages, severe pancreatitis often induces flatulence, intestinal dysfunction, intestinal flora translocation, and MODS. ${ }^{19}$ The three causes listed in this paragraph can possibly result in systemic infection, septic shock, and even MODS, thus increasing mortality rate.

In the patient grading process, we discovered that the evaluation of AGI grade caused difficulties to some extent. AGI grade I is a type of risk. For severe conditions, if patients can be cured with medicines, then the patients should be assigned to grade II. If the condition cannot be improved by medicines, then the patients should be assigned to grade III. Therefore, in the current study, diagnoses were complicated by time lags. To ascertain whether the patients' AGI grade could progress or be improved by medicines, we required time for further observation and judgment. On the other hand, heightened abdominal flatulence could be commonly observed in terminally ill patients. How could we evaluate this condition? For patients with exploratory surgery combined with severe shock, whether AGI was a complication of basic gastrointestinal disease, an adverse event from an operation or the result of shock usually could not be detected in clinical practice. Bladder pressure measurement is a significant tool used in diagnosing and predicting prognosis for abdominal compartment syndrome. ${ }^{20}$ However, only a few patients, most of whom were with severe pancreatitis, received bladder pressure measurement. Although there is still controversy over measuring bladder pressure for all critical patients, ${ }^{21}$ it is necessary to introduce this technology into clinical practice to help evaluate gastrointestinal function.

Erythromycin, metoclopramide, and domperidone were recommended by the ESICM WGAP. ${ }^{5}$ Recent research has discovered that 5-HT type 4 receptor agonist tegaserod cures small intestinal obstructions. ${ }^{22}$ In our current research, only one patient was prescribed with tegaserod to treat AGI; most of other patients were prescribed with mosapride, another type of 5-HT type 4 receptor agonist. Previous research pointed out that mitogen-activated protein kinases always participate in the expression of regularly tight junction proteins occludin and ZO-1 and injuries barrier function of intestinal epithelial cells. Mosapride may reverse these functions by inhibiting extracellular signal-regulated kinase- 1 and p38 protein phosphorylation. ${ }^{23,24}$ Mechanisms and effects of mosapride require further research. 
There were limitations to our current study. First, our present study was a retrospective study, and therefore we could only review and analyze the information of patients rather than improve the clinical outcomes. We could not search pathogenesis, either. Second, information we collected was simple, which could not be used for in-depth research on reasons or solutions for AGI. The current study relied on a cross-sectional survey and cohort study with ICU patients from 2010 to 2011 only. Nevertheless, in the present study, we discovered that the incidence rate of AGI was $46.11 \%$ in the ICU of the Shenzhen People's Hospital and that the mortality rate reached $34.74 \%$. The mortality rate increased with the increase of AGI grade. In our current study, we also discovered that AGI grade could be used as a prediction for mortality, and as a warning for clinicians. AGI grade may therefore be helpful to doctors in making clinical decisions. Third, we also found that the ESICM WGAP has some faults that should be resolved; therefore, in future studies, we will clarify these issues. Fourth, there are several factors that can affect the gastrointestinal tract, which may influence results in clinical practice. In future studies, we will perform some adjustments to decrease the influence of these factors.

\section{Conclusion}

In the present study, we concluded that secondary AGI caused by severe systemic conditions could result in worsened clinical outcomes. To the best of our knowledge, the current study is the first to reach this conclusion. Therefore, the 2012 ESICM WGAP recommendation are feasible and effective in guiding clinical practice except the lack of objective laboratory data. We recommend that the readers or doctors should apply this knowledge into the routine clinical practice.

\section{Acknowledgments}

The authors are sincerely grateful to Dr Yan Xia from Shenzhen Maritime Bureau for Statistic Analysis, Shenzhen, People's Republic of China, for the data. The authors also thank Dr JieYing Liao and LiYun Lin from the South China Normal University, Guangzhou, Guangdong, People's Republic of China, for their efforts to revise the paper.

\section{Disclosure}

The authors report no conflicts of interest in this work.

\section{References}

1. Stefaniak J, Baron DM, Metnitz PG, Kramer L. [Disturbances of gastrointestinal motility in intensive care units]. Anasthesiol Intensivmed Notfallmed Schmerzther. 2010;45:696-706. German.

2. Fruhwald S, Holzer P, Metzler H. [Gastrointestinal motility in acute illness]. Wien Klin Wochenschr. 2008;120:6-17. German.
3. Piton G, Manzon C, Cypriani B, Carbonnel F, Capellier G. Acute intestinal failure in critically ill patients: is plasma citrulline the right marker? Intensive Care Med. 2011;37:911-917.

4. Shimizu K, Ogura H, Hamasaki T, et al. Altered gut flora are associated with septic complications and death in critically ill patients with systemic inflammatory response syndrome. Dig Dis Sci. 2011;56:1171-1177.

5. Reintam Blaser A, Malbrain ML, Starkopf J, et al. Gastrointestinal function in intensive care patients: terminology, definitions and management. Recommendations of the ESICM Working Group on Abdominal Problems. Intensive Care Med. 2012;38:384-394.

6. Munro CL. The "lady with the lamp" illuminates critical care today. Am J Crit Care. 2010;19:315-317.

7. Williams JP, McBride WH. After the bomb drops: a new look at radiation-induced multiple organ dysfunction syndrome (MODS). Int J Radiat Riol. 2011;87:851-868.

8. Huang HH, Chang SJ, Hsu CW, Chang TM, Kang SP, Liu MY. Severity of illness influences the efficacy of enteral feeding route on clinical outcomes in patients with critical illness. J Acad Nutr Diet. 2012;112:1138-1146.

9. Sun JK, Li WQ, Ni HB, et al. Modified gastrointestinal failure score for patients with severe acute pancreatitis. Surg Today. 2013;43:506-513.

10. Haller D, Holt L, Parlesak A, et al. Differential effect of immune cells on non-pathogenic Gram-negative bacteria-induced nuclear factor-kappaB activation and pro-inflammatory gene expression in intestinal epithelial cells. Immunology. 2004;112:310-320.

11. Kalia N, Pockley AG, Wood RF, Brown NJ. Effects of FK409 on intestinal ischemia-reperfusion injury and ischemia-induced changes in the rat mucosal villus microcirculation. Transplantation. 2001;72:1875-1880.

12. $\mathrm{Du} \mathrm{J}, \mathrm{Hu} \mathrm{H}$, Liu R, et al. Clinical effects of intensive insulin therapy treating traumatic shock combined with multiple organ dysfunction syndrome. J Huangzhong Univ Sci Technolog Med Sci. 2011;31: 194-198.

13. Esfandyari T, Macnaughton WK, Quirion R, St Pierre S, Junien JL, Sharkey KA. A novel receptor for calcitonin gene-related peptide (CGRP) mediates secretion in the rat colon: implications for secretory function in colitis. FASEB J. 2000;14:1439-1446.

14. Baumgart DC, Dignass AU. Intestinal barrier function. Curr Op in Clin Nutr Metab Care. 2002;5:685-694.

15. Troeger H, Richter JF, Beutin L, et al. Escherichia coli alpha-haemolysin induces focal leaks in colonic epithelium: a novel mechanism of bacterial translocation. Cell Microbiol. 2007;9:2530-2540.

16. Hou H, Ping X, Zhu Y, Zhao Z, Li Y, Li J. Dietary fiber alleviates intestinal barrier dysfunction in post-trauma rats. Cin Invest Med. 2010; 33:E117.

17. Han H, Wang HL, Yu XZ, Guo SB. [Gastrointestinal dysfunction/failure and critical care medicine]. Zhongguo Yi Xue Ke Xue Yuan Xue Bao. 2008;30:224-227. Chinese.

18. Zhang Z, Xiang Y, Wang B, et al. Intestinal mucosal permeability of children with cefaclor-associated serum sickness-like reactions. Eur J Pediatr. 2013;172:537-543.

19. Wang G, Wen J, Xu L, et al. Effect of enteral nutrition and ecoimmunonutrition on bacterial translocation and cytokine production in patients with severe acute pancreatitis. J Surg Res. 2013;183:592-597.

20. Katsios C, Ye C, Hoad N, Piraino T, Soth M, Cook D. Intra-abdominal hypertension in the critically ill: interrater reliability of bladder pressure measurement. J Crit Care. 2013;28:e1-e6.

21. Starkopf J, Tamme K, Blaser AR. Should we measure intra-abdominal pressures in every intensive care patient? Ann Intensive Care. 2012; 2:S9.

22. Ramirez R, Zuckerman MJ, Hejazi RA, Chokhavatia S. Treatment of acute colonic pseudo-obstruction with tegaserod. Am J Med Sci. 2010; 339:575-576.

23. Oshima T, Miwa H, Joh T. Aspirin induces gastric epithelial barrier dysfunction by activating p38 MAPK via claudin-7. Am J Physiol Cell Physiol. 2008;295:C800-C806.

24. Gao X, Zhang ZY, Wu HL, et al. [Protective effect of mosapride on acute gastric mucosal lesions induced by aspirin in rats]. Chin J Gastroenterol. 2012;17:550-554. Chinese. 
Therapeutics and Clinical Risk Management

Dovepress

\section{Publish your work in this journal}

Therapeutics and Clinical Risk Management is an international, peerreviewed journal of clinical therapeutics and risk management, focusing on concise rapid reporting of clinical studies in all therapeutic areas outcomes, safety, and programs for the effective, safe, and sustained use of medicines. This journal is indexed on PubMed Central, CAS,
EMBase, Scopus and the Elsevier Bibliographic databases. The manuscript management system is completely online and includes a very quick and fair peer-review system, which is all easy to use. Visit http://www.dovepress.com/testimonials.php to read real quotes from published authors.

Submit your manuscript here: http://www.dovepress.com/therapeutics-and-clinical-risk-management-journal 